\title{
Approximate point joint spectra and multiplicative functionals
}

by

ANDRZEJ SOŁTYSIAK (Poznań)

Abstract. We show that a unital Banach algebra has a nonzero multiplicative linea functional whose kernel consists of joint left [respectively right] topological divisors of zero if and only if the left [right] approximate point spectrum $\tau_{l}\left(a_{1}, \ldots, a_{n}\right)\left[\tau_{r}\left(a_{1}, \ldots, a_{n}\right)\right]$ is nonempty for every finite set of elements $a_{1}, \ldots, a_{n}$ in the algebra.

\$1. Introduction. Let $A$ be a unital complex Banach algebra. The unit of $A$ will be denoted by 1 . The left approximate point spectrum of an $n$-tuple $\left(a_{1}, \ldots, a_{n}\right)$ of elements in $A$, denoted by $\tau_{1}^{A}\left(a_{1}, \ldots, a_{n}\right)$ or simply by $\tau_{1}\left(a_{1}, \ldots, a_{n}\right)$ if there is no confusion, is defined to be the subset of $C^{n}$ consisting of those $\left(\lambda_{1}, \ldots, \lambda_{n}\right)$ for which there exists a sequence $\left(u_{k}\right)$ of elements in $A$ such that $\left\|u_{k}\right\|=1$ for all $k$ and $\lim _{k}\left\|\left(a_{j}-\lambda_{j}\right) u_{k}\right\|=0$ for $j$ $=1, \ldots, n$. (Here, $a_{j}-\lambda_{j}$ stands for $a_{j}-\lambda_{j}$ 1.) The right approximate point spectrum $\tau_{r}\left(a_{1}, \ldots, a_{n}\right)$ is defined in a similar manner. The joint approximate point spectrum $\tau^{A}\left(a_{1}, \ldots, a_{n}\right)$, or simply written as $\tau\left(a_{1}, \ldots, a_{n}\right)$, is defined to be their union:

$$
\tau\left(a_{1}, \ldots, a_{n}\right)=\tau_{1}\left(a_{1}, \ldots, a_{n}\right) \cup \tau_{\mathrm{r}}\left(a_{1}, \ldots, a_{n}\right) .
$$

If $A=B(X)$ where $X$ is a complex Banach space, then

$$
\tau_{1}^{A}\left(a_{1}, \ldots, a_{n}\right)=\left\{\left(\lambda_{1}, \ldots, \lambda_{n}\right) \in C^{n}: \inf _{\|x\|=1} \sum_{j=1}^{n}\left\|\left(a_{j}-\lambda_{j}\right) x\right\|=0\right\}
$$

(and is usually denoted by $\sigma_{n}\left(a_{1}, \ldots, a_{n}\right)$, and called the "approximate point spectrum"), while

$$
\tau_{\mathrm{r}}^{A}\left(a_{1}, \ldots, a_{n}\right)=\left\{\left(\lambda_{1}, \ldots, \lambda_{n}\right) \in C^{n}: \sum_{j=1}^{n}\left(a_{j}-\lambda_{j}\right) X \neq X\right\}
$$

(and is usually denoted by $\sigma_{\mathrm{d}}\left(a_{1}, \ldots, a_{n}\right)$, and called the "defect spectrum"), (See [4], p. 95.)

Let us note that for an arbitrary $n$-tuple $\left(a_{1}, \ldots, a_{n}\right)$ of elements in $\boldsymbol{A}$ the following inclusions hold true:

$$
\tau_{1}^{A}\left(a_{1}, \ldots, a_{n}\right) \subset \sigma_{1}^{A}\left(a_{1}, \ldots, a_{n}\right), \quad \tau_{\mathrm{r}}^{A}\left(a_{1}, \ldots, a_{n}\right) \subset \sigma_{\mathrm{r}}^{A}\left(a_{1}, \ldots, a_{n}\right),
$$


where $\sigma_{1}^{A}\left(a_{1}, \ldots, a_{n}\right)$ and $\sigma_{\mathrm{r}}^{A}\left(a_{1}, \ldots, a_{n}\right)$ denote the left joint spectrum and the right joint spectrum of the $n$-tuple $\left(a_{1}, \ldots, a_{n}\right)$ respectively. (See [3] or [4] for their definitions.)

The approximate point spectra are always compact subsets of $C^{n}$. However, they can be void (see [4]). The main result of [3] says that if the joint spectrum $\sigma\left(a_{1}, \ldots, a_{n}\right)\left(=\sigma_{1}\left(a_{1}, \ldots, a_{n}\right) \cup \sigma_{\mathrm{r}}\left(a_{1}, \ldots, a_{n}\right)\right)$ is nonempty for an arbitrary $n$-tuple $\left(a_{1}, \ldots, a_{n}\right)$ of elements in $A$ with $n=1,2, \ldots$, then $A$ has a (nonzero) multiplicative (linear) functional. In view of the abovementioned inclusions the same result is true for the joint approximate point spectrum. Conversely, if $A$ has a multiplicative functional $\varphi$, then $\left(\varphi\left(a_{1}\right), \ldots, \varphi\left(a_{n}\right)\right) \in \sigma\left(a_{1}, \ldots, a_{n}\right)$ for an arbitrary $n$-tuple $\left(a_{1}, \ldots, a_{n}\right)$. However, this is not true for the joint approximate point spectrum. A suitable example will be given in the last section. It is clear that we have to assume something about this multiplicative functional. To explain this assumption we need the following concept.

Definition. A subset $S$ of a Banach algebra $A$ consists of joint left topological divisors of zero if for every finite subset $\left\{x_{1}, \ldots, x_{n}\right\}$ of $S$ there exists a sequence $\left(z_{k}\right)$ of elements in $A$ such that $\left\|z_{k}\right\|=1$ for every $k$ and $\lim _{k}\left\|x_{j} z_{k}\right\|=0$ for $j=1, \ldots, n$. The definition of a subset consisting of joint right topological divisors of zero is similar (cf. [8], p. 65). The fact that a subset $S$ of $A$ consists of joint left [respectively right] topological divisors of zero will be shortly denoted by $S \in l_{1}(A)\left[t_{\mathrm{r}}(A)\right]$.

Now, let us observe that, if $A$ has a multiplicative functional $\varphi$ with kernel consisting of joint left topological divisors of zero, then $\tau_{1}\left(a_{1}, \ldots, a_{n}\right)$ is always nonempty; in fact, in that case we have

$$
\left(\varphi\left(a_{1}\right), \ldots, \varphi\left(a_{n}\right)\right) \in \tau_{1}\left(a_{1}, \ldots, a_{n}\right) .
$$

Of course the same observation can be made for the right approximate point spectrum. fact:

The main purpose of the present paper is to show the converse of this

THEOREM. If $\tau_{1}\left(a_{1}, \ldots, a_{n}\right)$ [respectively $\tau_{\mathrm{r}}\left(a_{1}, \ldots, a_{n}\right)$ ] is nonempty for an arbitrary n-tuple $\left(a_{1}, \ldots, a_{n}\right)$ of elements in a Banach algebra $A$, with $n$ $=1,2, \ldots$, then $A$ has a multiplicative functional $\varphi$ with $\operatorname{ker} \varphi$ consisting of joint left [right] topological divisors of zero.

§ 2. Main results, We start with the following:

Lemma. If a function $\varphi: A \rightarrow C$ is such that

$$
(\varphi(x), \varphi(y), \varphi(z)) \in \tau_{1}(x, y, z)\left[\tau_{\mathrm{r}}(x, y, z)\right]
$$

for arbitrary elements $x, y, z$ in $A$, then it is linear and multiplicative.
Proof. We shall give the proof for the left approximate point spectrum. The argument for the right spectrum is analogous. To avoid brackets let us denote $\varphi(x)=\lambda_{x}$ for each $x$ in $A$. Now taking arbitrary $x, y$ in $A$ and $\alpha, \beta$ in $C$ we have $\left(\lambda_{\alpha x+\beta y}, \lambda_{x}, \lambda_{y}\right) \in \tau_{1}(\alpha x+\beta y, x, y)$. This implies that there exists a sequence $\left(z_{k}\right)$ such that $\left\|z_{k}\right\|=1$ for each $k$ and $\left(\alpha x+\beta y-\lambda_{a x+\beta y}\right) z_{k} \rightarrow 0$, $\left(x-\lambda_{x}\right) z_{k} \rightarrow 0,\left(y-\lambda_{y}\right) z_{k} \rightarrow 0$ as $k \rightarrow \infty$. We have

$$
\begin{aligned}
\mid \lambda_{\alpha x+\beta y} & -\alpha \lambda_{x}-\beta \lambda_{y}|=| \lambda_{\alpha x+\beta y}-\alpha \lambda_{x}-\beta \lambda_{y}|| z_{k} \| \\
& =\left\|\left(\lambda_{\alpha x+\beta y}-\alpha \lambda_{x}-\beta \lambda_{y}\right) z_{k}\right\| \\
& =\left\|\left(\lambda_{\alpha x+\beta y}-(\alpha x+\beta y)\right) z_{k}+\alpha\left(x-\lambda_{x}\right) z_{k}+\beta\left(y-\lambda_{y}\right) z_{k}\right\| \\
& \leqslant\left\|\left(\lambda_{\alpha x+\beta y}-(\alpha x+\beta y)\right) z_{k}\right\|+|\alpha|\left\|\left(x-\lambda_{x}\right) z_{k}\right\|+|\beta| \|\left(y-\lambda_{y}\right) z_{k} \mid .
\end{aligned}
$$

The right-hand side of this inequality tends to zero as $k \rightarrow \infty$, therefore $\lambda_{\alpha x+\beta y}=\alpha \lambda_{x}+\beta \lambda_{y}$ which means that the functional $\varphi$ is linear.

To see that it is multiplicative let us take arbitrary $x$ and $y$ in $A$. Since $\left(\lambda_{x y}, \lambda_{x}, \lambda_{y}\right) \in \tau_{1}(x y, x, y)$ there exists a sequence $\left(u_{k}\right)$ with $\left\|u_{k}\right\|=1$ for all $k$ and such that $\left(x y-\lambda_{x y}\right) u_{k} \rightarrow 0,\left(x-\lambda_{x}\right) u_{k} \rightarrow 0,\left(y-\lambda_{y}\right) u_{k} \rightarrow 0$ as $k \rightarrow \infty$. As before we have

$$
\begin{aligned}
\left|\lambda_{x y}-\lambda_{x} \lambda_{y}\right|=\mid \lambda_{x y}-\lambda_{x} \lambda_{y}\|\| u_{k}\|=\|\left(\lambda_{x y}-\lambda_{x} \lambda_{y}\right) u_{k} \| \\
\quad=\left\|\left(\lambda_{x y}-x y\right) u_{k}+x\left(y-\lambda_{y}\right) u_{k}+\lambda_{y}\left(x-\lambda_{x}\right) u_{k}\right\| \\
\leqslant\left\|\left(\lambda_{x y}-x y\right) u_{k}\left|+\|x\|\left\|\left(y-\lambda_{y}\right) u_{k}\right\|+\right| \lambda_{y} \mid\right\|\left(x-\lambda_{x}\right) u_{k} \| .
\end{aligned}
$$

Since the right-hand side of the above inequality tends to zero as $k \rightarrow \infty$ we have $\lambda_{x y}=\lambda_{x} \lambda_{y}$.

Remark. Using the Kowalski-Słodkowski generalization of the Gleason-Kahane-Żelazko theorem (see [5]) we can show that it is enough to take arbitrary two elements instead of three in the assumption of the lemma. Indeed, if we assume $(\varphi(x), \varphi(y)) \in \tau_{1}(x, y)$ for all $x$ and $y$ in $A$, then $(\varphi(0), \varphi(0)) \in \tau_{1}(0,0)=\{(0,0)\}$ which implies that $\varphi(0)=0$ and by the "oneway spectral mapping theorem" for $\tau_{1}$ (see [4], p. 100) we get $\varphi(x)$ $-\varphi(y) \in \tau_{1}(x-y) \subset \sigma(x-y)$. Hence by the Kowalski-Slodkowski theorem the functional $\varphi$ is linear and multiplicative. However, we gave the proof of the lemma with the assumption for three elements because it is very elementary while the proof of the Kowalski-Slodkowski theorem requires deep results on Lipschitz mappings in Fréchet spaces.

It should also be noted that the lemma is no longer true if we replace two by one element in the assumption (to see this it is enough to associate to each $2 \times 2$ complex matrix one of its eigenvalues).

Proof of the theorem. We assume that $\tau_{1}\left(a_{1}, \ldots, a_{n}\right)$ is always nonempty for finitely many elements $a_{1}, \ldots, a_{n}$ in $A$. Let an arbitrary $n$-tuple $\left(a_{1}, \ldots, a_{n}\right)$ be fixed from now on. For each $\stackrel{m}{m}$-tuple $\left(b_{1}, \ldots, b_{m}\right)$ of elements 
in $A$, we write $\varrho\left(b_{1}, \ldots, b_{m}\right)$ for the set of all $n$-tuples $\left(\lambda_{1}, \ldots, \lambda_{n}\right)$ in $C^{n}$ such that

$$
\left(\lambda_{1}, \ldots, \lambda_{n}, \mu_{1}, \ldots, \mu_{m}\right) \in \tau_{1}\left(a_{1}, \ldots, a_{n}, b_{1}, \ldots, b_{m}\right)
$$

for some $\left(\mu_{1}, \ldots, \mu_{m}\right) \in C^{m}$. By our assumption $\varrho\left(b_{1}, \ldots, b_{m}\right)$ is a nonempty compact subset of $C^{n}$. It is obvious that

$$
\varrho\left(b_{1}, \ldots, b_{m}, c_{1}, \ldots, c_{p}\right) \subset \varrho\left(b_{1}, \ldots, b_{m}\right) \cap \varrho\left(c_{1}, \ldots, c_{p}\right) .
$$

Therefore $\left\{\varrho\left(b_{1}, \ldots, b_{m}\right)\right\}$, where $\left(b_{1}, \ldots, b_{m}\right)$ runs through all $m$-tuples in $A^{m}$ and $m$ runs through all positive integers, is a family of compact sets with the finite intersection property, and hence its intersection is nonempty. Let $\tau_{1, \mu}\left(a_{1}, \ldots, a_{n}\right)$ denote this intersection, i.e. the set of all $\left(\lambda_{1}, \ldots, \lambda_{n}\right)$ in $C^{n}$ such that whenever $\left\{b_{1}, \ldots, b_{m}\right\}$ is a finite subset of $A,\left(\lambda_{1}, \ldots, \lambda_{n}, \mu_{1}, \ldots, \mu_{m}\right)$ $\in \tau_{1}\left(a_{1}, \ldots, a_{n}, b_{1}, \ldots, b_{m}\right)$ for some $\mu_{1}, \ldots, \mu_{m}$ in $\boldsymbol{C}$. It is a nonempty compact subset of $C^{n}$ (for an arbitrary $n$-tuple $\left(a_{1}, \ldots, a_{n}\right)$ in $A^{n}$ ).

Now, we shall show that $\tau_{1, \mu}$ has the following "projection property":

$$
P_{n}^{n+m} \tau_{1, \mu}\left(a_{1}, \ldots, a_{n}, a_{n+1}, \ldots, a_{n+m}\right)=\tau_{1, \mu}\left(a_{1}, \ldots, a_{n}\right)
$$

where $P_{n}^{n+m}$ is the canonical projection from $C^{n+m}$ onto $C^{n}$ which sends $\left(\lambda_{1}, \ldots, \lambda_{n}, \lambda_{n+1}, \ldots, \lambda_{n+m}\right)$ to $\left(\lambda_{1}, \ldots, \lambda_{n}\right)$. It is enough to prove this for $m=1$. The rest will follow by an easy induction argument. Let an arbitrary $(n+1)$-tuple $\left(a_{1}, \ldots, a_{n+1}\right)$ be fixed from now on. It is obvious that

$$
P_{n}^{n+1} \tau_{1, \mu}\left(a_{1}, \ldots, a_{n+1}\right) \subset \tau_{1, \mu}\left(a_{1}, \ldots, a_{n}\right) .
$$

To establish the converse inclusion let us take an aribtrary $\left(\lambda_{1}, \ldots, \lambda_{n}\right)$ from $\tau_{1, \mu}\left(a_{1}, \ldots, a_{n}\right)$. For each $m$-tuple $\left(b_{1}, \ldots, b_{m}\right)$ of elements in $A$ we write $\omega\left(b_{1}, \ldots, b_{m}\right)$ for the set of all complex numbers $\lambda$ such that

$$
\left(\lambda_{1}, \ldots, \lambda_{n}, \lambda, \mu_{1}, \ldots, \mu_{m}\right) \in \tau_{1}\left(a_{1}, \ldots, a_{n}, a_{n+1}, b_{1}, \ldots, b_{m}\right)
$$

for some $\left(\mu_{1}, \ldots, \mu_{m}\right) \in C^{m}$. The set $\omega\left(b_{1}, \ldots, b_{m}\right)$ is always nonempty since $\left(\lambda_{1}, \ldots, \lambda_{n}\right) \in \tau_{1, \mu}\left(a_{1}, \ldots, a_{n}\right)$. Moreover, it is easy to see that $\omega\left(b_{1}, \ldots, b_{m}\right)$ is compact and

$$
\omega\left(b_{1}, \ldots, b_{m}, c_{1}, \ldots, c_{p}\right) \subset \omega\left(b_{1}, \ldots, b_{m}\right) \cap \omega\left(c_{1}, \ldots, c_{p}\right)
$$

for all $b_{1}, \ldots, b_{m}, c_{1}, \ldots, c_{p}$ in $A$. So the family $\left\{\omega\left(b_{1}, \ldots, b_{m}\right)\right\}$, where $\left(b_{1}, \ldots, b_{m}\right)$ runs through all $m$-tuples in $A^{m}$ and $m$ runs through all positive integers, has the finite intersection property. Therefore its intersection is nonempty. Taking $\lambda_{n+1}$ from this intersection we get $\left(\lambda_{1}, \ldots, \lambda_{n}, \lambda_{n+1}\right)$ $\in \tau_{1, \mu}\left(a_{1}, \ldots, a_{n}, a_{n+1}\right)$.

To complete the proof of the theorem we shall use Kuratowski-Zorn's lemma. For this purpose let us denote by $\mathscr{P}$ the family of all ordered pairs.
$(X, \varphi)$, where $X$ is a (nonempty) subset of $A$ and $\varphi: X \rightarrow C$ is a function such that

$$
\left(\varphi\left(x_{1}\right), \ldots, \varphi\left(x_{n}\right)\right) \in \tau_{1, \mu}\left(x_{1}, \ldots, x_{n}\right)
$$

for every finite subset $\left\{x_{1}, \ldots, x_{n}\right\}$ of $X$. The family $\mathscr{P}$ is nonvoid (on each finite subset of $A$ one can construct a function with the above-mentioned property) and partially ordered by the following relation:

$$
\left(X_{1}, \varphi_{1}\right) \leqslant\left(X_{2}, \varphi_{2}\right) \quad \text { if and only if } \quad X_{1} \subset X_{2}
$$

and $\varphi_{2}$ restricted to $X_{1}$ coincides with $\varphi_{1}$.

It is standard that every totally ordered subfamily of $\mathscr{P}$ has an upper bound. Hence, by Kuratowski-Zorn's lemma $\mathscr{P}$ has a maximal element $\left(X_{0}, \varphi_{0}\right)$. We claim that $X_{0}=A$.

Suppose to the contrary that there exists an element $a$ in $A$ which does not belong to $X_{0}$. For an arbitrary $n$-tuple $\left(x_{1}, \ldots, x_{n}\right)$ of elements in $X_{0}$ we write $\delta\left(a, x_{1}, \ldots, x_{n}\right)$ for the set of all complex numbers $\lambda$ such that

$$
\left(\lambda, \varphi_{0}\left(x_{1}\right), \ldots, \varphi_{0}\left(x_{n}\right)\right) \in \tau_{1, \mu}\left(a, x_{1}, \ldots, x_{n}\right) .
$$

The projection property of $\tau_{1, \mu}$ implies that $\delta\left(a, x_{1}, \ldots, x_{n}\right)$ is always nonempty. It is obvious that it is compact and moreover

$$
\delta\left(a, x_{1}, \ldots, x_{n}, y_{1}, \ldots, y_{m}\right) \subset \delta\left(a, x_{1}, \ldots, x_{n}\right) \cap \delta\left(a, y_{1}, \ldots, y_{m}\right)
$$

for arbitrary elements $x_{1}, \ldots, x_{n}, y_{1}, \ldots, y_{m}$ in $X_{0}$ and all positive integers $n$, $m$. Thus the family $\left\{\delta\left(a, x_{1}, \ldots, x_{n}\right)\right\}$, where $\left(x_{1}, \ldots, x_{n}\right)$ runs through all $n$ tuples in $X_{0}^{n}$ and $n$ runs through all positive integers, has the finite intersection property. Let a number $\lambda$ be in the intersection of this family. Now, if define $\varphi(x)=\varphi_{0}(x)$ for $x$ in $X_{0}$ and $\varphi(a)=\lambda$, then the pair $\left(X_{0} \cup\{a\}, \varphi\right)$ belongs to $\mathscr{P}$. This contradicts the maximality of $\left(X_{0}, \varphi_{0}\right)$. Therefore $X_{0}=A$. Since $\tau_{1, \mu}\left(a_{1}, \ldots, a_{n}\right)$ is always contained in $\tau_{1}\left(a_{1}, \ldots, a_{n}\right)$ the lemma concludes the proof for the left approximate point spectrum. The proof for the right spectrum is similar.

Corollary 1. The joint approximate point spectrum $\tau\left(a_{1}, \ldots, a_{n}\right)$ is nonempty for every finite set $a_{1}, \ldots, a_{n}$ of elements in $A$ if and only if there exists on $A$ a multiplicative functional $\varphi$ such that $\operatorname{ker} \varphi$ consists of either joint left or joint right topological divisors of zero.

Proof. The "if" part is obvious. To prove the "only if" part let us assume that $\tau\left(a_{1}, \ldots, a_{n}\right) \neq \varnothing$ for an arbitrary $n$-tuple $\left(a_{1}, \ldots, a_{n}\right) \in A^{n}$ and each $n$. This means that always either $\tau_{1}\left(a_{1}, \ldots, a_{n}\right) \neq \varnothing$ or $\tau_{\mathrm{r}}\left(a_{1}, \ldots, a_{n}\right) \neq \varnothing$. If $\tau_{1}\left(a_{1}, \ldots, a_{n}\right)$ is always nonvoid we are done. If, on the other hand, $\tau_{1}\left(a_{1}, \ldots, a_{n}\right)=\varnothing$ for some elements $a_{1}, \ldots, a_{n}$ in $A$, then we have $\tau_{r}\left(a_{1}, \ldots, a_{n}\right) \neq \varnothing$. Actually, we then obtain $\tau_{r}\left(a_{1}, \ldots, a_{n}, b_{1}, \ldots, b_{m}\right)$ 
$\neq \varnothing$ for every $m$-tuple $\left(b_{1}, \ldots, b_{m}\right) \in A^{m}$ and each $m$, since if $\tau_{\mathrm{r}}\left(a_{1}, \ldots, a_{n 2}\right.$ $\left.b_{1}, \ldots, b_{m}\right)=\varnothing$ then $\tau_{1}\left(a_{1}, \ldots, a_{n}, b_{1}, \ldots, b_{m}\right) \neq \varnothing$, and the inclusion

$$
P_{n}^{n+m} \tau_{1}\left(a_{1}, \ldots, a_{n}, b_{1}, \ldots, b_{m}\right) \subset \tau_{1}\left(a_{1}, \ldots, a_{n}\right)
$$

implies that $\tau_{1}\left(a_{1}, \ldots, a_{n}\right) \neq \varnothing$, which is impossible. From this we can conclude that $\tau_{\mathrm{r}}\left(c_{1}, \ldots, c_{p}\right) \neq \varnothing$ for arbitrary elements $c_{1}, \ldots, c_{p}$ in $A$ and every $p$. .

From the proof of the theorem we have the following:

Corollary 2. For each n-tuple $\left(a_{1}, \ldots, a_{n}\right)$ of elements in $A$ and every positive integer $n$

$$
\tau_{1, \mu}\left(a_{1}, \ldots, a_{n}\right)=\left\{\left(\varphi\left(a_{1}\right), \ldots, \varphi\left(a_{n}\right)\right): \varphi\right. \text { is a multiplicative }
$$

$$
\text { functional on } A \text { with } \operatorname{ker} \varphi \in l_{1}(A) \text { \}. }
$$

COROLlaRY 3. The left approximate point spectrum $\tau_{1}$ has the projection property if and only if

$$
\tau_{1}\left(a_{1}, \ldots, a_{n}\right)=\left\{\left(\varphi\left(a_{1}\right), \ldots, \varphi\left(a_{n}\right)\right): \varphi\right. \text { is a multiplicative }
$$

functional on $A$ with ker $\left.\varphi \in l_{1}(A)\right\}$

for an arbitrary $n$-tuple $\left(a_{1}, \ldots, a_{n}\right) \in A^{n}$ and each positive integer $n$.

Proof. The projection property of $\tau_{1}$ implies that $\tau_{1}$ and $\tau_{1, \mu}$ coincide. So Corollary 2 gives the desired formula for $\tau_{1}$. The converse implication is obvious. -

It is evident that analogous results (to those of Corollaries 2 and 3) are true for the right approximate point spectrum.

COROLlaRY 4. The joint approximate point spectrum $\tau$ has the projection property if and only if

$$
\tau\left(a_{1}, \ldots, a_{n}\right)=\left\{\left(\varphi\left(a_{1}\right), \ldots, \varphi\left(a_{n}\right)\right): \varphi\right. \text { is a multiplicative }
$$

$$
\text { functional on } \left.A \text { with } \operatorname{ker} \varphi \in l_{1}(A) \cup l_{\mathrm{r}}(A)\right\}
$$

for an arbitrary n-tuple $\left(a_{1}, \ldots, a_{n}\right) \in A^{n}$ and each positive integer $n$.

Proof. Suppose that $\tau$ has the projection property and take arbitrary $n$ tuples $\left(a_{1}, \ldots, a_{n}\right) \in A^{n}$ and $\left(\lambda_{1}, \ldots, \lambda_{n}\right) \in \tau\left(a_{1}, \ldots, a_{n}\right)$. Without loss of generality we can assume that $\left(\lambda_{1}, \ldots, \lambda_{n}\right) \in \tau_{1}\left(a_{1}, \ldots, a_{n}\right)$. Then either $\left(\lambda_{1}, \ldots, \lambda_{n}\right) \in \tau_{1, \mu}\left(a_{1}, \ldots, a_{n}\right)$ or $\left(\lambda_{1}, \ldots, \lambda_{n}\right) \notin \tau_{1, \mu}\left(a_{1}, \ldots, a_{n}\right)$. If the first possibility occurs, then, by Corollary 2 , there exists a multiplicative functional $\varphi$ on $A$ such that its kernel consists of joint left topological zero divisors and $\varphi\left(a_{1}\right)=\lambda_{1}, \ldots, \varphi\left(a_{n}\right)=\lambda_{n}$. So assume that $\left(\lambda_{1}, \ldots, \lambda_{n}\right) \notin \tau_{1, \mu}\left(a_{1}, \ldots, a_{n}\right)$. Then there exists an $m$-tuple $\left(b_{1}, \ldots, b_{m}\right)$ of elements in $A$ such that

$$
\left(\lambda_{1}, \ldots, \lambda_{n}, \mu_{1}, \ldots, \mu_{m}\right) \notin \tau_{1}\left(a_{1}, \ldots, a_{n}, b_{1}, \ldots, b_{m}\right)
$$

for arbitrary complex numbers $\mu_{1}, \ldots, \mu_{m}$. The projection property of $\tau$ implies that there exist scalars $\lambda_{n+1}, \ldots, \lambda_{n+m}$ such that

$$
\left(\lambda_{1}, \ldots, \lambda_{n}, \lambda_{n+1}, \ldots, \lambda_{n+m}\right) \in \tau_{\mathrm{r}}\left(a_{1}, \ldots, a_{n}, b_{1}, \ldots, h_{m}\right) .
$$

Moreover, for an arbitrary $p$-tuple $\left(c_{1}, \ldots, c_{p}\right)$ of elements in $A$ we have

$$
\begin{aligned}
& \left(\lambda_{1}, \ldots, \lambda_{n}, \lambda_{n+1}, \ldots, \lambda_{n+m}, \mu_{1}, \ldots, \mu_{p}\right) \\
& \quad \in \tau_{r}\left(a_{1}, \ldots, a_{n}, b_{1}, \ldots, b_{m}, c_{1}, \ldots, c_{p}\right)
\end{aligned}
$$

for some $\left(\mu_{1}, \ldots, \mu_{p}\right) \in C^{n}$ (since otherwise we would get

$$
\left(\lambda_{1}, \ldots, \lambda_{n}, \lambda_{n+1}, \ldots, \lambda_{n+m}\right) \in \tau_{1}\left(a_{1}, \ldots, a_{n}, b_{1}, \ldots, b_{m}\right)
$$

which is impossible). This means that

$$
\left(\lambda_{1}, \ldots, \lambda_{n}, \lambda_{n+1}, \ldots, \lambda_{n+m}\right) \in \tau_{r, \mu}\left(a_{1}, \ldots, a_{n}, b_{1}, \ldots, b_{m}\right),
$$

and as before there exists a multiplicative functional $\varphi$ on $A$ with kernel consisting of joint right topological divisors of zero and such that $\varphi\left(a_{1}\right)$ $=\lambda_{1}, \ldots, \varphi\left(a_{n}\right)=\lambda_{n}$.

It is evident that if for every finite set $\left\{a_{1}, \ldots, a_{n}\right\}$ we have

$\tau\left(a_{1}, \ldots, a_{n}\right)=\left\{\left(\varphi\left(a_{1}\right), \ldots, \varphi\left(a_{n}\right)\right): \varphi\right.$ is a multiplicative functional on $A$ with $\left.\operatorname{ker} \varphi \in l_{1}(A) \cup l_{\mathrm{r}}(A)\right\}$,

then $\tau$ has the projection property.

Remark. If the algebra $A$ is commutative, then always $\tau_{1}\left(a_{1}, \ldots, a_{n}\right)$ $=\tau_{r}\left(a_{1}, \ldots, a_{n}\right)=\tau\left(a_{1}, \ldots, a_{n}\right)$, and by a result of M.-D. Choi, Ch. Davis, Z. Słodkowski and W. Żelazko (see [1] and [7]) the approximate point spectrum has the projection property. In this case we obviously have $\tau\left(a_{1}, \ldots, a_{n}\right)=\left\{\left(\varphi\left(a_{1}\right), \ldots, \varphi\left(a_{n}\right)\right): \varphi\right.$ is a multiplicative functional on $A$ with $\operatorname{ker} \varphi$ consisting of joint topological zero divisors\}.

By a result of V. Müller (see [6]) a multiplicative functional has kernel consisting of joint topological divisors of zero if and only if it can be extended to a multiplicative functional on every superalgebra of $A$. The set of such functionals is called the cortex of the algebra $A$, in symbols cor $A$.

Thus we see that

$$
\tau\left(a_{1}, \ldots, a_{n}\right)=\left\{\left(\varphi\left(a_{1}\right), \ldots, \varphi\left(a_{n}\right)\right): \varphi \in \operatorname{cor} A\right\}
$$

for each $n$-tuple $\left(a_{1}, \ldots, a_{n}\right)$ of elements in a commutative Banach algebra $A$ and every positive integer $n$.

On the other hand, if the left approximate point spectrum $\tau_{1}$ has the projection property on $A$ then the algebra $A / \operatorname{rad} A$ must be commutative (where $\operatorname{rad} A$ stands for the radical of $A$ ). Indeed, by Corollary 3 we have $\tau_{1}((a b-b a) c)=\{0\}$ for arbitrary elements $a, b, c$ in $A$. Since $\partial \sigma((a b$ $-b a) c) \subset \tau_{1}((a b-b a) c)$ (see [4], p. 103 or [8], p. 57) we have $\sigma((a b-b a) c)$ 
$=\{0\}$, and so $a b-b a \in \operatorname{rad} A$. The same observation can be made for the spectra $\tau_{\mathrm{r}}$ and $\tau$.

We do not know any example of a noncommutative Banach algebra on which the left [right] aproximate point spectrum has the projection property. Hence we conjecture that if it is so, then the algebra must be commutative. An example given at the end of the paper will show that the commutativity of the algebra modulo its radical does not suffice for the approximate point spectra $\tau_{1}, \tau_{\mathrm{r}}$, and $\tau$ to have the projection property (while it is a necessary and sufficient condition for the joint spectrum $\sigma$, and also for $\sigma_{1}$ and $\sigma_{\mathrm{r}}$, to have such property; see [3]).

\$. Examples. In this final section we give the previously announced examples.

EXAMPLE 1. It will be shown that an algebra can have multiplicative functionals but the joint approximate point spectrum of some $n$-tuples can be empty. The idea of this example is due to C.-K. Fong (see [2]).

Let $A$ be the algebra $M_{5}(C)$ of all $5 \times 5$ matrices with complex entries. Take the following two elements of $A$ :

$$
a_{1}=\left[\begin{array}{lllll}
0 & 1 & 0 & 0 & 0 \\
0 & 0 & 0 & 0 & 0 \\
0 & 0 & 0 & 1 & 0 \\
0 & 0 & 0 & 0 & 1 \\
0 & 0 & 0 & 0 & 0
\end{array}\right], \quad \text { and } \quad a_{2}=\left[\begin{array}{lllll}
0 & 0 & 1 & 0 & 0 \\
1 & 0 & 0 & 0 & 0 \\
0 & 0 & 0 & 0 & 0 \\
0 & 0 & 0 & 0 & 0 \\
0 & 0 & 0 & 1 & 0
\end{array}\right] .
$$

Then we have $a_{1}^{3}=a_{2}^{3}=0$. Hence $\sigma^{A}\left(a_{1}\right)=\sigma^{A}\left(a_{2}\right)=\{0\}$. This implies that $\sigma^{A}\left(a_{1}, a_{2}\right) \subset\{(0,0)\}$ (cf. [4], p. 92). But if we take

$$
a_{3}=\left[\begin{array}{rrrrr}
0 & 0 & 0 & 0 & 0 \\
1 & 0 & 0 & -1 & 0 \\
0 & 0 & 0 & 0 & 0 \\
0 & 0 & 1 & 0 & 0 \\
0 & 0 & 0 & 0 & 0
\end{array}\right], \quad \text { and } \quad a_{4}=\left[\begin{array}{rrrrr}
0 & 1 & 0 & 0 & -1 \\
0 & 0 & 0 & 0 & 0 \\
1 & 0 & 0 & 0 & 0 \\
0 & 0 & 0 & 0 & 1 \\
0 & 0 & 0 & 0 & 0
\end{array}\right],
$$

then we shall get $a_{1} a_{3}+a_{2} a_{1}=1$ (identity matrix) and $a_{2} a_{1}+a_{4} a_{2}=1$. Therefore $\sigma^{A}\left(a_{1}, a_{2}\right)=\varnothing$. Now, let us take $B$ to be the subalgebra of $A$ generated by $a_{1}, a_{2}$, and 1 . If we assign to each element $b$ of $B$ the entry of $b$ in the third row and the third column we shall get a multiplicative functional $\varphi$ on $B$. Since $\varphi\left(a_{1}\right)=\varphi\left(a_{2}\right)=0$, we get $(0,0) \in \sigma^{B}\left(a_{1}, a_{2}\right)$. However, we have $\tau^{B}\left(a_{1}, a_{2}\right) \subset \tau^{A}\left(a_{1}, a_{2}\right) \subset \sigma^{A}\left(a_{1}, a_{2}\right)$ (see [4], p. 92). Therefore $\tau^{B}\left(a_{1}, a_{2}\right)=\varnothing$
Example 2. Now we describe an algebra in which the joint approximate point spectrum is always nonempty but it does not have the projection property. entries

Let $A$ be the algebra of all $3 \times 3$ upper triangular matrices with complex

$$
a=\left[\begin{array}{ccc}
a(11) & a(12) & a(13) \\
0 & a(22) & a(23) \\
0 & 0 & a(33)
\end{array}\right] .
$$

It is known (see [4], pp. 94-95) that for all $a_{1}, \ldots, a_{n}$ in $A$ we have the equality $\sigma\left(a_{1}, \ldots, a_{n}\right)=\left\{\left(a_{1}(j j), \ldots, a_{n}(j j)\right): j=1,2,3\right\}$, while $\left(a_{1}(11), \ldots, a_{n}(11)\right)$ $\in \tau_{1}\left(a_{1}, \ldots, a_{n}\right)$ and $\left(a_{1}(33), \ldots, a_{n}(33)\right) \in \tau_{r}\left(a_{1}, \ldots, a_{n}\right)$. Hence $\tau_{1}\left(a_{1}, \ldots, a_{n}\right)$, $\tau_{\mathbf{r}}\left(a_{1}, \ldots, a_{n}\right)$, and $\tau\left(a_{1}, \ldots, a_{n}\right)$ are always nonvoid. However, if we take for example

$$
a=\left[\begin{array}{lll}
2 & 1 & 0 \\
0 & 1 & 1 \\
0 & 0 & 3
\end{array}\right] \text { and } b=\left[\begin{array}{lll}
2 & 2 & 0 \\
0 & 1 & 3 \\
0 & 0 & 3
\end{array}\right]
$$

then it is a matter of easy computations to show that $\tau_{1}(a, b)=\{(2,2)\}$, $\tau_{\mathrm{r}}(a, b)=\{(3,3)\}$ and $\tau_{1}(a)=\tau_{\mathrm{r}}(a)=\{2,1,3\}$. Thus $(1,1) \notin \tau(a, b)$ which means that the spectra $\tau_{1}, \tau_{\mathrm{r}}$, and $\tau$ do not have the projection property on this algebra.

Let us also note that the algebra $A$ is commutative modulo the radical.

It may be interesting to observe that on the algebra of all $2 \times 2$ upper triangular matrices the joint approximate point spectrum coincides with the joint spectrum, and therefore it has the projection property, while the left and right approximate point spectra do not have this property.

\section{References}

[1] M.-D. Choi and Ch. Davis, The spectral mapping theorem for joint approximate point spectrum, Bull. Amer. Math. Soc. 80 (1974), 317 321.

[2] C.-K. Fong, Multiplicative functionals and jolnt spectra, mimeographed notes of a lecture presented to the Seminar on Operator Theory, University of Toronto, November 1983 (unpublished).

[3] C.-K. Fong and. A. Soltysiak, Existence of a multiplicative functional and joint spectra, Studia Math. 81 (1985), 213-220.

[4] R. E. Harte, Spectral mapping theorems, Proc. Roy. Irish Acad. Sect. A 72 (1972), 89-107.

[5] S. Kowalski and Z. Slodk owski, A characterization of multiplicative linear functionals in Banach algebras, Studia Math. 67 (1980), 215 -223. 
[6] V. Muller, Non-removable ideals in commutative Banach algebras, ibid. 74 (1982), 97-104

[7] Z. Stodk owski and W. Żelazko, On joint spectra of commuting families of operators, ibid. 50 (1974), 127-148.

[8] W. Żelazko, Banach Algebras, PWN, Warszawa 1973.

INSTYTUT MATEMATYKI UNIWERSYTETU im. ADAMA MICKIEWICZA INSTITUTE OF MATHEMATICS, A. MICKIEWICZ UNIVERSITY

Matejki 48/49, 60-769 Poznań, Poland

Added in proof (May 1987). V. Muller pointed out to the author an example of a noncommutative Banach algebra for which the left and right approximate point spectra have the projection property. Hence the conjecture on p. 284 is false (see the author's forthcoming paper On the projection property of approximate point joint spectra, Comment. Math., vol. 28).

\section{Note on a theorem by Reshetnyak-Gurov}

by

INGEMAR WIK (Umeå)

Abstract. The paper gives a sharp estimate for the $L^{p}$-norm of functions whose mean oscillation in every cube is at most a fixed multiple, $\varepsilon$, of the mean value of the function in that cube. The estimate improves an earlier result of Reshetnyak-Gurov as $\varepsilon \rightarrow 0$.

In their paper [2] Reshetnyak and Gurov study functions with a mean oscillation which in every cube is not greater than a fixed multiple of the mean value of the function in that particular cube. Their result has been used by Bojarski [1] in a study of the stability of inverse Hölder inequalities.

A cube in $\boldsymbol{R}^{n}$ will always mean a cube with sides parallel to the axes. We let $|E|$ denote the Lebesgue measure of the set $E$ and prove the following theorem:

THEOREM. Let $q$ be any positive number, $\varepsilon$ a number in the range $0<\varepsilon$ $<\left(3 \cdot 2^{1 / q}\right)^{-1}$ and $f$ a vector-valued function $f: \Omega \rightarrow \boldsymbol{R}^{m}, \Omega \subset \boldsymbol{R}^{n}$. Suppose that for every cube $Q$ in $\Omega$ there exists a vector $f_{Q}$ in $\boldsymbol{R}^{m}$ such that

$$
\frac{1}{|Q|} \int_{Q}\left|f(x)-f_{Q}\right|^{q} d x \leqslant \varepsilon^{q}\left|f_{Q}\right|^{q} .
$$

Then $f$ has to be a function in $L_{100}^{p}(\Omega)$ for $q \leqslant p<c_{1} \varepsilon^{-1}$. For these values of $p$ we have for every cube $Q$ in $\Omega$

$$
\int_{Q}\left|f(x)-f_{Q}\right|^{p} d x<c_{2}\left|f_{Q}\right|^{p-q} \varepsilon^{p-q} \int_{Q}\left|f(x)-f_{Q}\right|^{q} d x
$$

$c_{1}$ may be taken as $(q \ln 2)\left(6 \cdot 2^{n+1 / q}\right)^{-1}$ and $c_{2}$ depends only on $p, q$ and $n$.

Remark 1. This constitutes an improvement of the result in [2] in that it contains a factor $\varepsilon \log (a(q) / \varepsilon)$ instead of $\varepsilon$ on the right-hand side of (2) and also requires $q$ to be at least 1 .

Remark 2. It is easy to find an example showing that (2) gives the best possible order as $\varepsilon$ tends to zero.

Proof. Let $Q$ be an arbitrary cube in $\Omega$ and put

$$
E_{1}=\left\{x \in Q ;\left|f(x)-f_{Q}\right|>\delta\left|f_{Q}\right|\right\},
$$

